\title{
The gluino-glue particle and relevant scales for the simulations of supersymmetric Yang-Mills theory
}

\author{
Georg Bergner* \\ University of Frankfurt, Institute for Theoretical Physics \\ E-mail: G.bergnerdphysik.uni-frankfurt.de \\ Istvan Montvay \\ Deutsches Elektronen-Synchrotron DESY, \\ Notkestr. 85, D-22603 Hamburg, Germany
}

Gernot Münster, Dirk Sandbrink, Umut D. Özugurel

University of Münster, Institute for Theoretical Physics

Wilhelm-Klemm-Strasse 9, D-48149 Münster, Germany

\begin{abstract}
Supersymmetric Yang-Mills theory is in several respects different from QCD and pure Yang-Mills theory. Therefore, a reinvestigation of the scales, at which finite size effects and lattice artifacts become relevant, is necessary. Both, finite size effects and lattice artifacts, induce a breaking of supersymmetry. In view of the unexpected mass gap between bosonic and fermionic particles an estimation of these effects is essential.
\end{abstract}

The 30th International Symposium on Lattice Field Theory

June 24 - 29, 2012

Cairns, Australia

${ }^{*}$ Speaker. 


\section{Introduction}

Supersymmetry plays a central role in theoretical models for elementary particle physics beyond the Standard Model. Therefore it is important to gain knowledge about the properties of supersymmetric theories. Much of what is known about supersymmetric models is based on treelevel considerations or comes from perturbation theory. However, various important characteristics, like the existence of mass-degenerate supermultiplets of particles, are of a non-perturbative nature. Therefore it is desirable to study them by means of non-perturbative methods.

The simplest supersymmetric model including gauge fields is the supersymmetric Yang-Mills theory (SYM). It describes interacting gluons and their supersymmetric partners, the gluinos, which are Majorana fermions in the adjoint representation of the gauge group $\mathrm{SU}\left(N_{c}\right)$. The (on-shell) Lagrangian of SYM in Minkowski space is

$$
\mathscr{L}=\operatorname{Tr}\left[-\frac{1}{4} F_{\mu v} F^{\mu v}+\frac{\mathrm{i}}{2} \bar{\lambda} \gamma^{\mu} D_{\mu} \lambda-\frac{m_{g}}{2} \bar{\lambda} \lambda\right],
$$

where $\lambda$ is the gluino field, $A_{\mu}$ the gluon field, $F_{\mu \nu}$ the non-Abelian field strength, and

$$
D_{\mu} \lambda^{a}=\partial_{\mu} \lambda^{a}+g f_{a b c} A_{\mu}^{b} \lambda^{c}
$$

denotes the gauge covariant derivative in the adjoint representation. The gluino mass term breaks supersymmetry softly.

SYM is similar to QCD in some respect [四]. It is asymptotically free and is assumed to show confinement. The "physical" particles are bound states of gluons and gluinos, and if supersymmetry is unbroken, they would form supermultiplets. The non-perturbative properties one would like to investigate with the lattice simulations include: (1) the spontaneous breaking of chiral symmetry, $Z_{2 N_{c}} \rightarrow Z_{2}$, that manifests itself in the non-vanishing vacuum expectation value $\langle\lambda \lambda\rangle \neq 0$, (2) the confinement of static quarks, indicated by a linear rise in the static quark potential, which is an evidence for the confining nature of the theory, (3) the spectrum of bound states, that can be compared to the predictions of low energy effective actions, (4) whether supersymmetry is spontaneously broken, (5) the restoration of SUSY in the continuum limit of a lattice regularisation.

The particle content of SYM is expected to consist of colour neutral bound states of gluons and gluinos, forming supermultiplets. Based on effective Lagrangeans, it has been predicted [, B] that the low-lying particles form two chiral supermultiplets, each consisting of a scalar, a pseudoscalar, and a fermionic spin 1/2 particle. One of them contains a $0^{-}$gluinoball (a- $\eta^{\prime} \sim \bar{\lambda} \gamma_{5} \lambda$ ), a $0^{+}$gluinoball $\left(\mathrm{a}-f_{0} \sim \bar{\lambda} \lambda\right)$, and a spin $1 / 2$ gluino-glueball $\left(\chi \sim \sigma^{\mu v} \operatorname{Tr}\left(F_{\mu v} \lambda\right)\right.$ ), the other one a $0^{-}$glueball, a $0^{+}$glueball, and a gluino-glueball. Both supermultiplets contain an exotic particle state called gluino-glueball, which is a spin 1/2 Majorana fermion. Such a bound state containing a single fermion does not occur in QCD, but analogous particles exist in models similar to QCD with an arbitrary number of quark flavours in the adjoint representation.

We study SYM non-perturbatively in the framework of regularisation on a lattice. Supersymmetry is generically broken in any non-trivial theory on the lattice [䧃]. The particle spectrum and the supersymmetric Ward identities can show how it is restored in the continuum limit or whether there is a possible remnant breaking of SUSY by non-perturbative effects. The existence of supermultiplets is an important signal for the supersymmetric limit of the theory. 
Previous work by our collaboration on SYM on the lattice, concerning the nonperturbative items mentioned above, is reported in [ [5, 目] and references given there. The results obtained there have not yet shown the expected degeneracy of the fermionic and bosonic masses. The mass of the gluino-glueball appeared to be larger than the other masses of its lightest possible superpartners. However, the masses were obtained at a fixed lattice spacing and without a detailed analysis of the finite size effects. Below we shall discuss our recent calculations, which indicate that the influence of the finite lattice spacing is larger than expected and provides a possible source of the supersymmetry breaking in the simulation.

\section{SUSY on the Lattice}

In our work we employ the formulation of SYM on a lattice by Curci and Veneziano [D], where the gluinos are described by Wilson fermions in the adjoint representation. SYM has also been investigated with domain wall fermions [ [8, Q, 미] and overlap fermions [ए]], which, however, require significantly more computing resources than Wilson fermions for large lattice volumes and small lattice spacings.

The lattice action of SYM in our setup is

$$
S_{L}=\beta \sum_{p}\left(1-\frac{1}{N_{c}} \operatorname{Re} \operatorname{Tr} U_{p}\right)+\frac{1}{2} \sum_{x y} \bar{\lambda}_{x}(\mathrm{D})_{x y} \lambda_{y},
$$

where $\mathrm{D}$ is the Wilson-Dirac operator

$$
\begin{aligned}
(\mathrm{D})_{x, a, \alpha ; y, b, \beta}= & \delta_{x y} \delta_{a, b} \delta_{\alpha, \beta} \\
& -\kappa \sum_{\mu=1}^{4}\left[\left(1-\gamma_{\mu}\right)_{\alpha, \beta}\left(V_{\mu}(x)\right)_{a b} \delta_{x+\mu, y}+\left(1+\gamma_{\mu}\right)_{\alpha, \beta}\left(V_{\mu}^{\dagger}(x-\mu)\right)_{a b} \delta_{x-\mu, y}\right]
\end{aligned}
$$

and $\left(V_{\mu}(x)\right)_{a b}$ are the gauge link variables in the adjoint representation. We are currently considering the gauge group $\mathrm{SU}(2)$ with generators $T^{a}=\tau_{a} / 2$, in which case

$$
\left(V_{\mu}(x)\right)_{a b}=2 \operatorname{Tr}\left(U_{x \mu}^{\dagger} T_{a} U_{x \mu} T_{b}\right) .
$$

The hopping parameter $\kappa$ is related to the bare gluino mass via $\kappa=1 /\left(2 m_{g}+8\right)$.

In order to reduce lattice artifacts, in our simulations we actually use the tree-level Symanzik improved gauge action, and one level of stout smearing applied to the link fields in the WilsonDirac operator.

From considerations of chiral and SUSY Ward identities it is expected $[\square]$ that a fine-tuning of the bare gluino mass parameter (i.e. $\kappa$ ) in the continuum limit is sufficient to approach the chiral symmetry and supersymmetry of the continuum theory. This tuning is most efficiently done by means of the mass of the unphysical adjoint pion $(a-\pi)$. This particle is the pion in the corresponding theory with two Majorana fermions in the adjoint representation. The correlator of this particle is the connected contribution of the $a-\eta^{\prime}$ correlator. The a- $\pi$ is not a physical particle in SYM. However, it can be defined in a partially quenched setup, in the same way as for one-flavour QCD []]. On the basis of arguments involving the OZI-approximation of SYM [], the adjoint pion 
mass is expected to vanish for a massless gluino. The corresponding value of $\kappa_{c}$ is most easily obtained from the dependence of the a- $\pi$-mass on $\kappa$.

The numerical simulations are done using a polynomial hybrid Monte Carlo (PHMC) algorithm with a two step polynomial approximation and reweighting [[13], []]. The functional integral over Majorana fermions yields the Pfaffian $\operatorname{Pf}(C D)=\operatorname{sign}(\operatorname{Pf}(C D)) \sqrt{\operatorname{det}(C D)}$ of the antisymmetric fermion matrix multiplied by the charge conjugation matrix $C$. The PHMC implements the positive weight $\sqrt{\operatorname{det}(\mathrm{D})}$, while the sign $\operatorname{sign}(\operatorname{Pf}(C \mathrm{D}))$ is taken into account by reweighting. The reweighting factors are obtained from the number of negative real eigenvalues of $\mathrm{D}$.

\section{The particle spectrum on the lattice}

The most interesting particles are the possible candidates for the lowest lying multiplets. These multiplets consist of a fermionic, a scalar, and a pseudoscalar particle. On the lattice a mixing between possible operators representing the corresponding quantum numbers is expected. As representations of the bosonic scalar and pseudoscalar particles we have measured glueballs and gluinoballs. The fermionic particle is represented by the gluino-glueball.

The correlation function of the $0^{++}$glueball is calculated by smeared plaquette-plaquette correlations. Variational smearing techniques (APE and HYP smearing) are used to get a reasonable signal for the particle mass. Unfortunately the signal for the $0^{-+}$glueball turned out to be insufficient for a determination of the mass with our current statistics.

The gluinoballs are similar to flavour singlet mesonic operators in QCD. Hence they contain disconnected contributions and require the computation of all-to-all propagators. In a graphical representation of the correlators the disconnected contributions show up as two disconnected fermion loops, e. g. for the $a-\eta^{\prime}$

$$
\left\langle\bar{\lambda}(x) \gamma_{5} \lambda(x) \bar{\lambda}(y) \gamma_{5} \lambda(y)\right\rangle=\left\langle\bigcap_{x}-2{ }^{x} \bigcup^{y}\right\rangle .
$$

From the computation of flavour singlet mesons in QCD it is well known that the all-to-all propagators introduce additional statistical noise in the computation of an observable. We observe this effect in our simulations when we compare the larger statistical errors of the $a-\eta^{\prime}$ with the small error of the a- $\pi$. We have implemented and tested several techniques to determine the all-to-all propagators [14]]. In our recent simulations a combination of the truncated eigenmode approximation and the stochastic estimator method has been used. Our truncated eigenmode approximation is implemented in such a way that from the eigenvalues reweighting factors can be obtained to improve the polynomial approximation of the PHMC algorithm. The stochastic estimator method was improved with iterations of a truncated solver, see [ㄷ]] for the details of these methods. With these techniques the $\mathrm{a}-\eta^{\prime}$ mass can be obtained with a reasonable precision, while the determination of the a $-f_{0}$ mass is still not satisfactory.

In contrast to the difficulties in the bosonic sector, the mass of the fermionic gluino-glueball can been obtained most reliably. The corresponding lattice operator is $\sigma^{\mu \nu} \operatorname{Tr}\left[F_{\mu \nu} \lambda\right]$, where the field strength on the lattice is represented by the clover plaquette. A combination of APE (applied on $F_{\mu v}$ ) and Jacobi smearing (applied on $\lambda$ ) leads to a further reduction of the noise for this signal. Plots of the effective masses for this particles can be found in Fig. . 


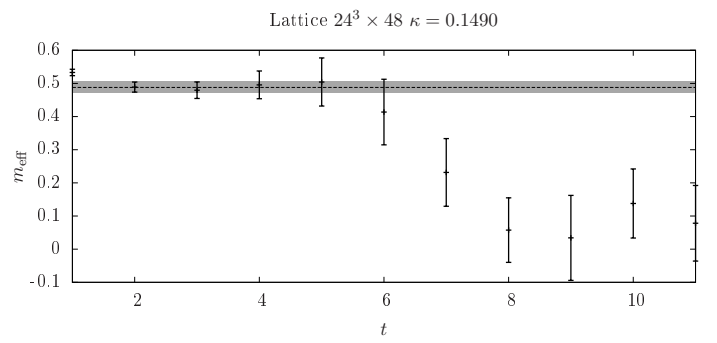

(a) $0^{+}$glueball

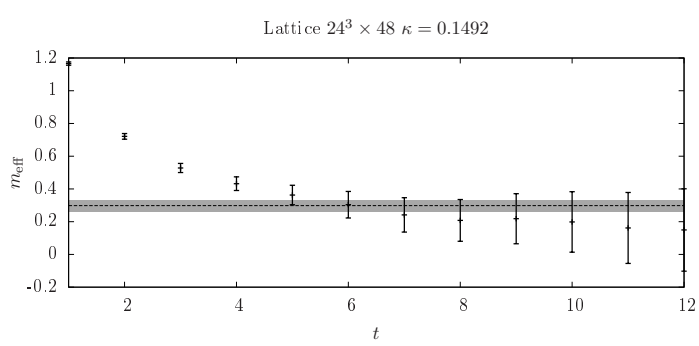

(c) $\mathrm{a}-f_{0}$

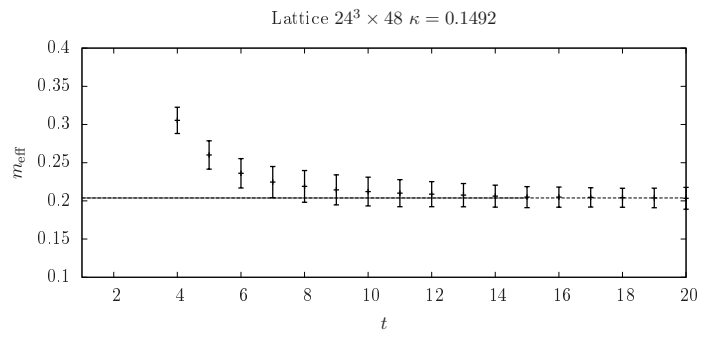

(e) $a-\pi$

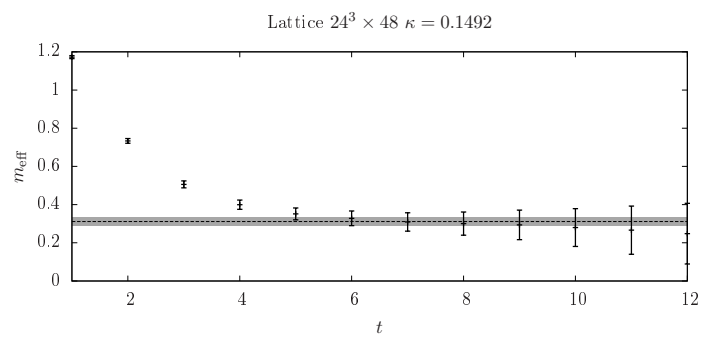

(b) $\mathrm{a}-\eta^{\prime}$

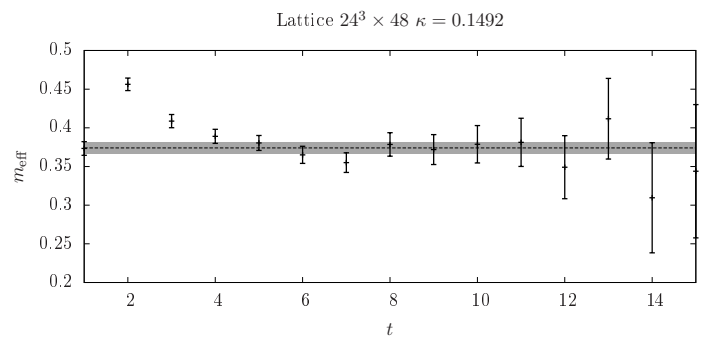

(d) gluino-glue

Figure 1: Examples for the effective masses of the particles measured in supersymmetric Yang-Mills theory. Time slice distances $t, t+a$ are used to compute the effective mass $m_{\mathrm{eff}}$ at distance $t$ assuming a single cosh (sinh) behavior. The mass and $t$ are given in units of the lattice spacing $a$. The lattice size is $24^{3} \times 48$. The parameters of the simulations are $\beta=1.75$ and $\kappa=0.1490 / 0.1492$.

\section{Finite size effects and lattice artifacts}

In previous work of our collaboration a rather large gap between fermionic and bosonic masses has been observed [5]. These simulations were done at $\beta=1.6$ and lattice sizes up to $24^{3} \times 48$. It would, however, be premature to draw conclusions from this about possible supersymmetry breakings in continuum SYM. There are several limits involved in the determination of physical results for this model, namely the infinite volume limit, the continuum limit, and the chiral limit, which have to be taken in this order. With present resources it is not possible to carry out this program. In recent simulations we have, however, been able to obtain some estimates for the relevance and influence of these limits.

We have performed a detailed investigation of the finite size effects in simulations of $8^{3} \times 16$, $12^{3} \times 24,16^{3} \times 36,20^{3} \times 40,24^{3} \times 48$, and $32^{3} \times 64$ lattices at $\beta=1.75$ [ए]]. For the asymptotic dependence of masses on the box size $L$ we have taken [ㅁ], [18]

$$
m(L) \approx m_{0}+C L^{-1} \exp \left(-\alpha m_{0} L\right)
$$




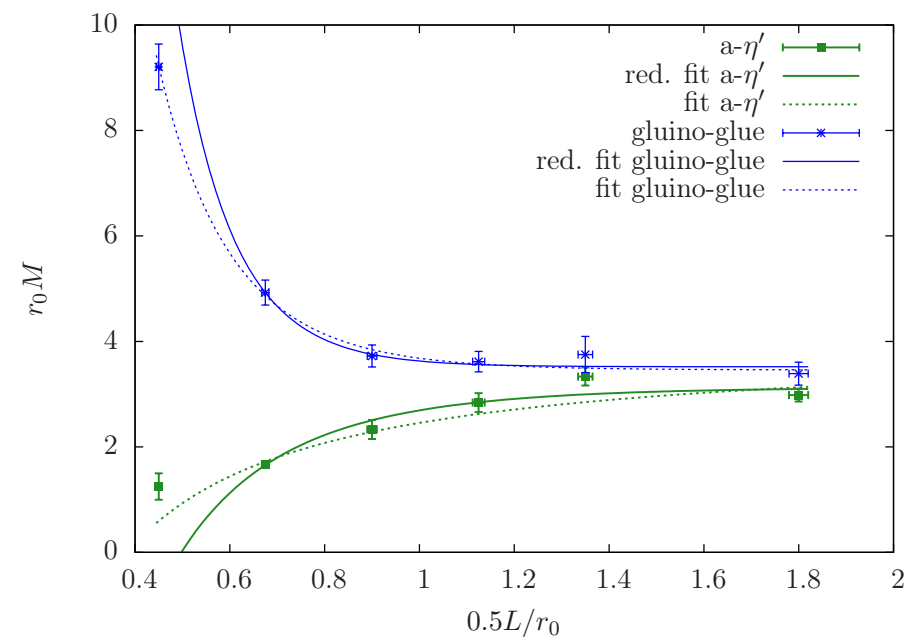

Figure 2: The influence of the finite box size $L$ on the mass gap between the bosonic a $-\eta^{\prime}$ and the fermionic gluino-glueball. These results were obtained at a fixed value of the bare parameter $\kappa=0.1490(\beta=1.75)$. The mass is, as indicated, shown in units of $r_{0}^{-1}$, and $L$ in units of $r_{0} / 0.5$, where $r_{0}$ is the Sommer parameter. When $r_{0}$ is set to its QCD value, the units of $L$ would correspond to fm.

for large values of $L$. Results have been obtained at fixed bare parameter $\kappa$ and at a fixed mass of $a-\pi$. As explained above, the best signal is obtained for the masses of the gluino-glueball and the $\mathrm{a}-\eta^{\prime}$. The difference between their masses can be taken as an estimate of the mass gap between the particles of a supermultiplet. The dependence of the mass gap on the finite box size is shown in Fig. [.

The mass gap is clearly increased by the influence of the finite volume. The supersymmetry breaking terms, that are present due to the lattice discretisation are hence increased by the finite size of the lattice. However, this effect becomes rather small already at moderately large lattice sizes. Therefore, finite size effects cannot be the source of the observed SUSY-breaking effects.

We have also been able to estimate the influence of the finite lattice spacing from simulations at a second larger value of $\beta$. The difference between the results at $\beta=1.6(a \approx 0.08 \mathrm{fm})$ and $\beta=1.75(a \approx 0.06 \mathrm{fm})$ are shown in Fig. [3. They indicate that indeed the lattice artifacts are the most relevant source for a bias in the spectrum of the particles.

Possible alternative ways to reduce the lattice artifacts are currently being explored. For example, an increased smearing in the fermionic part of the action seems to reduce their influence.

\section{Recent results}

The results of our investigations of finite size effect and lattice artifacts are the basis for our current simulations. The influence of the finite volume seems to be small. A small lattice spacing is important to keep the influence of the lattice artifacts under control. This result, obtained from the gluino-glue and the a- $\eta^{\prime}$ mass, is already quite convincing. For a complete investigations of the effects it is, however, indispensable to get information also form the other particle states. To distinguish the small mass gap at $\beta=1.75$ from the statistical and systematic errors, a good signal 


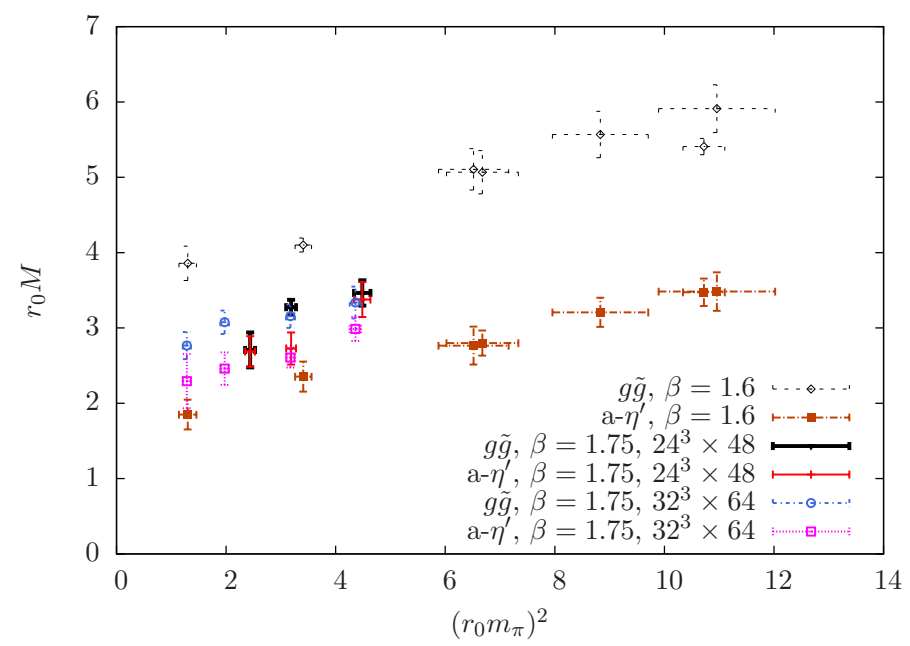

Figure 3: The mass of the $\mathrm{a}-\eta^{\prime}$ and the gluino-glueball at two different $\beta$, where the larger value corresponds to the smaller lattice spacing. The masses are shown as a function of the squared mass of the adjoint pion $\left(m_{\pi}\right)$. The results from the two different lattice sizes $24^{3} \times 48$ and $32^{3} \times 64$ are found to be consistent. All masses are given in units of the Sommer parameter $r_{0}$.

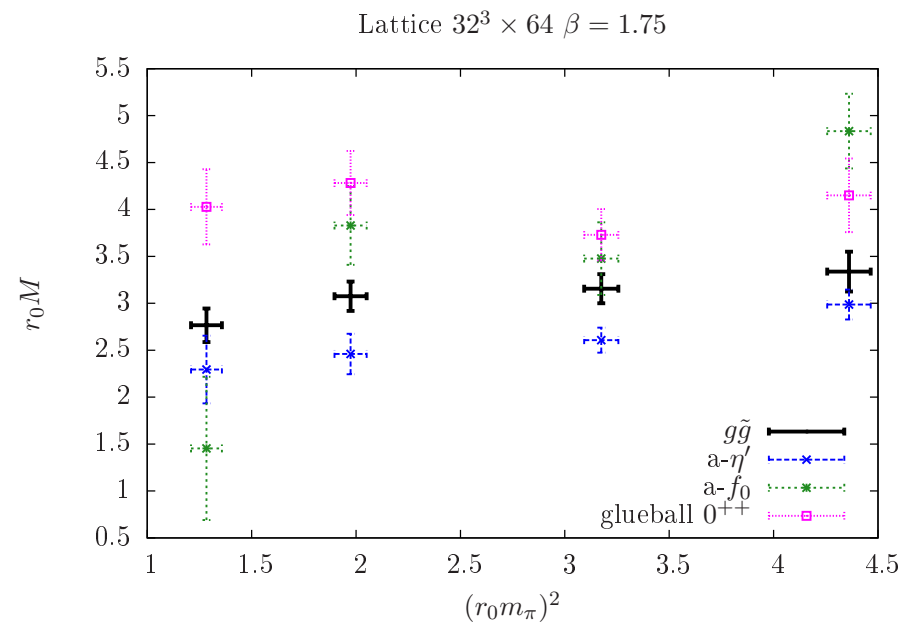

Figure 4: The masses of all particles at $\beta=1.75(a \approx 0.06 \mathrm{fm})$ obtained on a $32^{3} \times 64$ lattice $(L=1.8 \mathrm{fm})$ with a statistics of about 5000 configurations at each point. All masses are given in units of $r_{0}$.

is needed for all observables. Before we test further improvements of the action to reduce the lattice artifacts we have, therefore, made improvements in the measurement of the glueballs and the scalar mesons. Especially for the glueballs most important for an improvement is to increase the statistics significantly. Our first investigations were done at a lattice size of $32^{3} \times 64(L=1.8 \mathrm{fm})$, see Fig. 团. In our investigations of the finite size effects we found that these results are consistent with those at a lattice size of $24^{3} \times 48(L=1.35 \mathrm{fm})$. Based on these findings we have chosen the smaller lattice size and increased the statistics from about 5000 configurations at each $\kappa$ to around 10000. A preliminary summary of these data is shown in Fig. [5. 


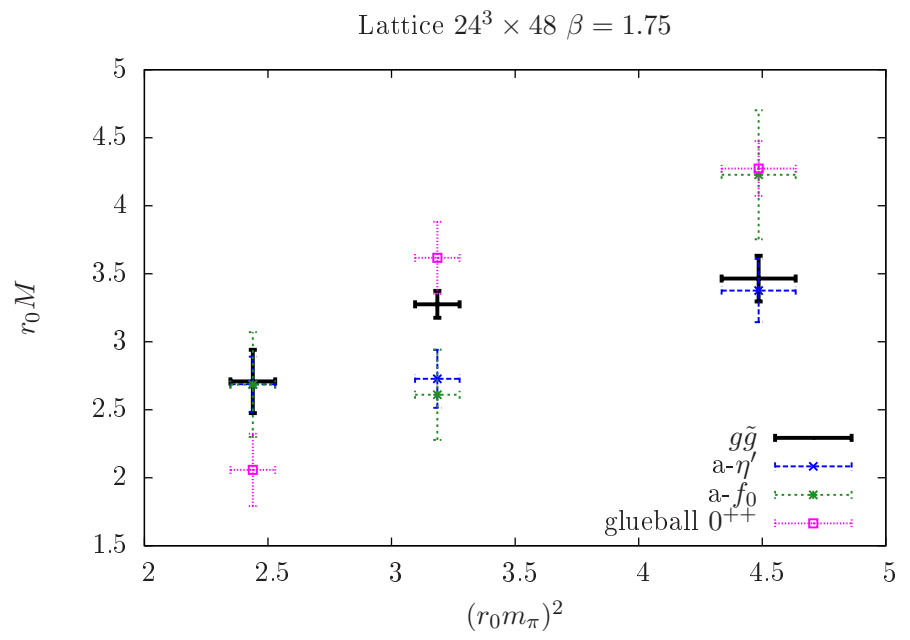

Figure 5: The masses of all particles at $\beta=1.75(a \approx 0.06 \mathrm{fm})$ obtained on a $24^{3} \times 48$ lattice $(L=1.35 \mathrm{fm})$ with a statistics of about 10,000 configurations at each point. All masses are given in units of $r_{0}$.

\section{Conclusions}

We have investigated the influence of the various simulation parameters on the unexpectedly observed mass gap between the particle states of a supersymmetric multiplet.

The finite size effects increase the mass gap as found from the splitting of the a- $\eta^{\prime}$ and gluinoglueball mass. The supersymmetry breaking terms that are introduced by the lattice discretisation seem to get finite size corrections that enlarge their influence. However, this effect is found to be small compared to the statistical error at moderate lattice sizes above around $1.2 \mathrm{fm}$.

The finite lattice spacing was the most relevant source of supersymmetry breaking in the previous results of our collaboration. The mass gap at our current, smaller, lattice spacing is significantly reduced compared to the previous results. In view of the statistical errors of the observables, it is already difficult to obtain the mass gap.

To determine the remaining mass gap with enough precision and to answer the question, whether it persists in the continuum limit, it is necessary to reduce the statistical errors of the observables. One important parameter for such an improvement is the statistics. An investigation of the improvements of the observables is the main task of our current simulations.

Improvements of the action, like an increased level of stout smearing or the inclusion of a clover term might also help to get a more precise answer about the relevance of the mass gap in the continuum limit. We have started to investigate these improvements.

\section{Acknowledgments}

This project is supported by the German Science Foundation (DFG) under contract Mu 757/16, and by the John von Neumann Institute for Computing (NIC) with grants of computing time. Further computing time has been provided by the computer cluster PALMA of the University of Münster. 


\section{References}

[1] D. Amati, K. Konishi, Y. Meurice, G. C. Rossi, G. Veneziano, Phys. Rept. 162 (1988) 169.

[2] G. Veneziano, S. Yankielowicz, Phys. Lett. B 113 (1982) 231.

[3] G. R. Farrar, G. Gabadadze, M. Schwetz, Phys. Rev. D 58 (1998) 015009 [arXiv:hep-th/971166].

[4] G. Bergner, JHEP 1001 (2010) 024 [arXiv:0909.4791 hep-lat]].

[5] K. Demmouche, F. Farchioni, A. Ferling, I. Montvay, G. Münster, E. E. Scholz, J. Wuilloud, Eur. Phys. J. C 69 (2010) 147 [arXiv:1003.2073 [hep-lat]].

[6] G. Bergner, I. Montvay, G. Münster, D. Sandbrink, U. D. Özugurel, PoS(Lattice 2011) 055 [arXiv:[11.3012 [hep-lat]].

[7] G. Curci, G. Veneziano, Nucl. Phys. B 292 (1987) 555.

[8] G. T. Fleming, J. B. Kogut and P. M. Vranas, Phys. Rev. D 64 (2001) 034510 [arXiv:hep-lat/0008009].

[9] J. Giedt, R. Brower, S. Catterall, G. T. Fleming and P. Vranas, Phys. Rev. D 79 (2009) 025015 [arXiv:0810.5746 [hep-lat]].

[10] M. G. Endres, Phys. Rev. D 79 (2009) 094503 [arXiv:0902.4267 [hep-lat]].

[11] S. W. Kim et al. [JLQCD Collaboration], PoS (Lattice 2011) 069 [arXiv:M11.2180 hep-lat]].

[12] F. Farchioni, I. Montvay, G. Münster, E. E. Scholz, T. Sudmann, J. Wuilloud, Eur. Phys. J. C 52 (2007) 305 [arXiv:0706.1131 hep-lat]].

[13] I. Montvay, E. Scholz, Phys. Lett. B 623 (2005) 73 [arXiv:hep-lat/0506006].

[14] F. Farchioni, G. Münster, R. Peetz, Eur. Phys. J. C 38 (2004) 329 [arXiv:hep-lat/0404004].

[15] G. S. Bali, S. Collins, A. Schäfer, Comput. Phys. Commun. 181 (2010) 1570 [arXiv:0910.3970 [hep-lat]].

[16] G. Bergner, T. Berheide, I. Montvay, G. Münster, U. D. Özugurel, D. Sandbrink, JHEP 1209 (2012) 108 [arXiv:[206.2341 hep-lat]].

[17] M. Lüscher, Commun. Math. Phys. 104 (1986) 177.

[18] G. Münster, Nucl. Phys. B 249 (1985) 659. 